\title{
Phenomena in Inverse Stackelberg Games, Part 2: Dynamic Problems
}

\author{
G.J. Olsder
}

Published online: 28 May 2009

(C) The Author(s) 2009. This article is published with open access at Springerlink.com

\begin{abstract}
Dynamic two-person games are considered, in which the roles of the players are hierarchical. One player behaves as a leader, the other one as a follower. Such games are named after Stackelberg. In the current paper, a special type of such games is considered, known in the literature as inverse Stackelberg games. In such games, the leader announces his strategy as a mapping from the follower's decision space into his own decision space. Arguments for studying such problems are given. This paper specifically studies dynamic games, i.e. the underlying model is described by an ordinary differential equation. The decisions of both players have a time component. As in the static case, the routine way of analysis, leading to a study of composed functions, is not very fruitful. Other approaches are given, mainly by studying specific examples.
\end{abstract}

Keywords Differential games · Transaction costs $\cdot$ Stackelberg games · Inverse Stackelberg games $\cdot$ Composed functions

\section{Problem Setting and Terminology}

This paper deals with dynamic inverse Stackelberg problems and is a follow-up of [1], which dealt with static inverse Stackelberg problems. Such problems are usually treated within the context of game theory. In the simplest form, there are two players, called leader and follower respectively, each having its own cost function,

$$
J_{\mathrm{L}}\left(u_{\mathrm{L}}, u_{\mathrm{F}}\right), J_{\mathrm{F}}\left(u_{\mathrm{L}}, u_{\mathrm{F}}\right),
$$

Communicated by Y.-C. Ho.

G.J. Olsder $(\bowtie)$

Faculty of Electrical Engineering, Mathematics and Computer Science, Delft University

of Technology, P.O. Box 5031, 2600 GA Delft, The Netherlands

e-mail: g.j.olsder@tudelft.nl 
where $u_{\mathrm{L}}, u_{\mathrm{F}} \in \mathrm{R}$. Each player wants to choose its own decision variable in such a way as to minimize its own cost function. Without giving an equilibrium concept, the problem as stated so far is not well defined. Such an equilibrium concept could for instance be one named after Nash or after Pareto [2]. In this paper, the inverse Stackelberg equilibrium as it was introduced in [3], will be considered. The leader announces a function $\gamma_{\mathrm{L}}(\cdot)$ which maps $u_{\mathrm{F}}$ into $u_{\mathrm{L}}$. Examples of games with such informatiom structures are:

- Think of the leader being the government and of the follower as a citizen. The government states how much income tax the citizen has to pay and this tax will depend on the income $u_{\mathrm{F}}$ of the citizen. It is up to the citizen as to how much money to earn (by working harder or not) and thus he can choose $u_{\mathrm{F}}$. The income tax the government will receive equals $\gamma_{\mathrm{L}}\left(u_{\mathrm{F}}\right)$, where the "rule for taxation" $\gamma_{\mathrm{L}}(\cdot)$, in many countries piecewise linear, was made known ahead of time.

- The leader is the bank and the follower the investor. The investor can buy stocks, with the bank as intermediator, with the money he has in his savings account. Suppose he buys stocks worth $u_{\mathrm{F}}$ Euros. Then the bank will charge him $\gamma_{\mathrm{L}}\left(u_{\mathrm{F}}\right)$ as transaction costs. The function $\gamma_{\mathrm{L}}(\cdot)$ has been made known by the bank before the actual transaction takes place $[4,5]$.

Given the function $\gamma_{\mathrm{L}}(\cdot)$, the follower will make his choice $u_{\mathrm{F}}$ according to

$$
u_{\mathrm{F}}^{*}=\arg \min _{u_{\mathrm{F}}} J_{\mathrm{F}}\left(\gamma_{\mathrm{L}}\left(u_{\mathrm{F}}\right), u_{\mathrm{F}}\right) .
$$

Subsequently the decision of the leader, who is supposed to stick to what he promised, is then $u_{\mathrm{L}}^{*}=\gamma_{\mathrm{L}}\left(u_{\mathrm{F}}^{*}\right)$. Optimizing quantities will be provided with an asterix. The leader, before announcing his $\gamma_{\mathrm{L}}(\cdot)$, will of course realize how the follower will play and he should exploit this knowledge in order to choose the best possible $\gamma$-function, such that ultimately his own cost function $J_{\mathrm{L}}$ becomes as small as possible. Symbolically, we could write

$$
\gamma_{\mathrm{L}}^{*}(\cdot)=\arg \min _{\gamma_{\mathrm{L}}(\cdot)} J_{\mathrm{L}}\left(\gamma_{\mathrm{L}}\left(u_{\mathrm{F}}\left(\gamma_{\mathrm{L}}(\cdot)\right)\right), u_{\mathrm{F}}\left(\gamma_{\mathrm{L}}(\cdot)\right)\right)
$$

In this way, one enters the field of composed functions [6], which is known to be a notoriously complex area.

In [1], we dealt with problems in which each of the players had to make one decision only. Sometimes such problems are called static. In the current paper, extensions are considered in which the players must act more often, either in a discrete time or a continuous time setting, and such problems are called dynamic. In the latter case, one is usually given a model,

$$
\dot{x}=f\left(x, u_{\mathrm{L}}, u_{\mathrm{F}}\right), \quad x(0)=x_{0},
$$

through which the players interact. The state $x$ of this model evolves in time (indicated by $t$ ) according to the differential equation $\dot{x}=f$. The dot on a variable refers to the time derivative, i.e. $\dot{x}=\frac{\mathrm{d} x}{\mathrm{~d} t}$. Generally the state is an $n$-dimensional vector (written as $x \in \mathrm{R}^{n}$ ), but in this paper we will confine ourselves to $n=1$, and it will be considered on the interval $0 \leq t \leq T$, where $T$ represents a fixed final time. The 
vector $x_{0}$ is the initial condition. The quantities $u_{i}, i=\mathrm{L}, \mathrm{F}$, are scalar functions of time possibly restricted to a certain set: $u_{i}(t) \in U_{i}(t)$. The function $u_{i}$ must be chosen in such a way as to minimize the cost function

$$
J_{i}\left(u_{\mathrm{L}}, u_{\mathrm{F}}\right)=\int_{0}^{T} g_{i}\left(x, u_{1}, u_{2}\right) \mathrm{d} t+q_{i}(x(T)) ;
$$

both $g_{i}$ and $q_{i}$ are scalar functions and are assumed to satisfy certain regularity conditions such that the cost functions are well defined. Under suitable conditions on the function $f$, the time evolution of $x$ is uniquely determined by the differential equation. There are no restrictions on $x(T)$; we deal with so-called free endpoint problems. With respect to the equilibrium concept, various extensions to the static situation exist, even within a leader-follower context; see [2] for some possibilities.

In [1], some references with respect to "classic", i.e. static, Stackelberg games were given. Some references with respect to the more recently developed dynamic Stackelberg game theory are [5] and [7-9].

The further content of the paper is as follows. In Sect. 2, the main problem is formulated. In Sects. 3 and 4, two dynamic examples are considered and it is shown what the difficulties are in obtaining the solutions. The mathematically oriented reader should be warned somewhat that, at this moment, only little theory is available (with for instance theorems about existence, uniqueness), and that the theory is still in its infancy by discovering phenomena by means of examples.

\section{Problem Statement}

The main problem to be considered is

$$
\begin{aligned}
\dot{x} & =f(x, u), \quad x(0)=x_{0}, \\
\min _{u} J_{\mathrm{F}}^{\mathrm{c}} & =\min _{u}\left(q(x(T))+\int_{0}^{T} g(x, u) \mathrm{d} t+\int_{0}^{T} \gamma(u(t)) \mathrm{d} t\right), \\
\max _{\gamma(\cdot)} J_{\mathrm{L}} & =\max _{\gamma(\cdot)} \int_{0}^{T} \gamma(u(t)) \mathrm{d} t .
\end{aligned}
$$

The notation has been changed somewhat here. Instead of $u_{\mathrm{F}}$, we write simply $u$ for the decision variable of the follower. The function $\gamma$ is up to the choice of the leader (we do not write $u_{\mathrm{L}}$ anymore) subject to the restriction

$$
\gamma(0)=0, \quad \gamma(\cdot) \geq 0
$$

Occasionally, we will also require that $\gamma$ is nondecreasing with respect to $|u|$. Also note that the cost function of the follower, including the integral term with the $\gamma$-function, is indicated by $J_{\mathrm{F}}^{\mathrm{c}}$ (the notation $J_{\mathrm{F}}$ will be reserved for this cost function without the integral term containing the $\gamma$-function). The inverse Stackelberg equilibrium should be recognized easily by means of the notation: the leader announces the function $\gamma$ which thus becomes known to the follower who subsequently chooses $u$. 
A possible interpretation of this model is that the follower is an investor who wants to maximize his wealth,

$$
-q(x(T))-\int_{0}^{T} g(x, u) \mathrm{d} t,
$$

equivalently wants to minimize his loss $J_{\mathrm{F}} \stackrel{\text { def }}{=} q(x(T))+\int_{0}^{T} g(x, u) \mathrm{d} t$. Please note the difference in the notations $J_{\mathrm{F}}^{\mathrm{c}}$ and $J_{\mathrm{F}}$. The term $-q(x(T))$ in the criterion represents the wealth of the investor at the final time $T$ and the term $-\int_{0}^{T} g(x, u) \mathrm{d} t$ represents the consumption during the time interval $[0, T]$. The decision variable $u(t)$ denotes the transactions with the bank at time $t$ (e.g. selling or buying stocks). To be more precise, $u(t)$ denotes a transaction density, i.e. during the time interval $[t, t+\mathrm{d} t]$ the number of transactions equals $u(t) \mathrm{d} t$. For $u=0$, no transaction takes place $(\gamma(0)=0)$. Such transactions cost money and we assume that the bank wants to maximize these transaction costs as indicated by (3). These costs are added to the costs of the follower as indicated in (2). Another reasonable restriction on $\gamma$ is that $\gamma(u)$ is nondecreasing with respect to $|u|$. The higher the number of transactions (either buying or selling, one being related to a positive $u$, the other one to a negative $u)$, the higher the costs.

In the sections to come, it will become clear that the main problem as stated is a difficult one. In [5], a variational approach, starting from the Hamiltonian $H$ in the minimum principle formulation of Pontryagin [10], which lead to a condition containing composed functions [6], was suggested without much success.

As a direct extension of Theorem 2.1 from [1], we have that an upper bound for the profit of the bank is $J_{\mathrm{F}}(u \equiv 0)-J_{\mathrm{F}}\left(u=u^{*}\right)$, where $J_{\mathrm{F}}$ is the follower's pure cost function, i.e. without the additional banking costs and where $u^{*}$ minimizes this pure cost function (of course subject to the state equation (1)). In [5], the following conjecture was posed.

Conjecture 2.1 Generic sufficiency conditions for the bank to have its profit arbitrarily close to the upper bound given above (this property is referred to as incentive controllability in [2]) are either one of the following:

- The integrand $\gamma$ is allowed to depend explicitly on $t$, i.e. $\gamma(t, u(t))$;

- $\gamma$ depends on $u$ only and $u^{*}$ is constant.

This conjecture is false as will be shown by the examples to come.

\section{First Dynamic Example}

The model is

$$
\dot{x}=u, \quad x(0)=1,
$$

and the criterion is $\min _{u} J_{\mathrm{F}}$, where

$$
J_{\mathrm{F}}=\left(\frac{1}{2} \int_{0}^{1} u^{2}(t) \mathrm{d} t+\frac{1}{2} x^{2}(1)\right) .
$$


In this optimal control problem, the integral term in the cost function is simply part of this cost function; it is not to be considered as transaction costs (transaction costs will be added shortly). The optimal solution is obtained through the following formulas:

$$
\begin{aligned}
& H=\lambda u+\frac{1}{2} u^{2} \rightarrow u^{*}=-\lambda, \\
& \dot{\lambda}=0, \quad \lambda(1)=x(1), \quad u(t)=-x(1), \quad t \in[0,1],
\end{aligned}
$$

and hence,

$$
u^{*}(t)=-\frac{1}{2}, \quad x^{*}(t)=1-\frac{1}{2} t, \quad J_{\mathrm{F}}\left(u=u^{*}\right)=\frac{1}{4}, \quad J_{\mathrm{F}}(u=0)=\frac{1}{2} .
$$

The notation $H$ stands for Hamiltonian. The minimum principle of Pontryagin dictates that $H$ must be minimized with respect to $u$.

The above optimal control problem is now extended to a game problem by adding transaction costs. The criterion is changed into $\min _{u}^{\mathrm{c}} J_{\mathrm{F}}$, where

$$
J_{\mathrm{F}}^{\mathrm{c}}=\left(\frac{1}{2} \int_{0}^{1} u^{2}(t) \mathrm{d} t+\frac{1}{2} x^{2}(1)+\int_{0}^{1} \gamma(u(t)) \mathrm{d} t\right) .
$$

The function $\gamma$ satisfies the by now usual restrictions $\gamma(\cdot) \geq 0, \gamma(0)=0$. There is another criterion for the second player: $\max _{\gamma(\cdot)} J_{\mathrm{L}}$, where

$$
J_{\mathrm{L}}=\int_{0}^{1} \gamma(u(t)) \mathrm{d} t
$$

\subsection{Ad Hoc Approach}

A likely candidate for the optimal $\gamma$ is $\gamma(u)=-\left(\frac{1}{2}-\epsilon\right) u(1+u)$ on the interval $[-1,0]$ and $\gamma(u) \geq 0$ elsewhere, with $\epsilon$ an arbitrarily small positive number. (This choice of $\gamma$ mimics the idea for the first choice of $\gamma$ in Example 2.1 of [1].) Here $\gamma$ is, if $\epsilon$ would be zero, equal to $-H$ on the essential interval. We now find

$$
\begin{aligned}
H & =\lambda u+\frac{1}{2} u^{2}-\left(\frac{1}{2}-\epsilon\right) u(1+u) \rightarrow u^{*}=-\frac{\lambda-\frac{1}{2}+\epsilon}{2 \epsilon}, \\
\dot{\lambda} & =0, \quad \lambda(1)=x(1), \\
x^{*}(t) & =1-\frac{1}{2} t, \quad J_{\mathrm{F}}^{\mathrm{c}}=\frac{3}{8}-\frac{1}{4} \epsilon, \quad J_{\mathrm{L}}=\frac{1}{8}-\frac{1}{4} \epsilon .
\end{aligned}
$$

Note that, with this choice of $\gamma$, if it is optimal, Conjecture 2.1 does not hold.

The bank can do better, however, even with a quadratic $\gamma$. Let us try

$$
\gamma(u)=\frac{1}{2} \beta u^{2}+\alpha u
$$


on a certain interval to be determined. Automatically $\gamma(0)=0$. Under the condition $\beta>-1$, it is straightforward to show that

$$
x^{*}(1)=\frac{1+\beta-\alpha}{2+\beta}, \quad u^{*}(t) \equiv-\frac{1+\alpha}{2+\beta} .
$$

Since $J_{\mathrm{F}}(u=0)=\frac{1}{2}$, the parameters $\alpha$ and $\beta$ must necessarily satisfy $J_{\mathrm{F}}^{\mathrm{c}}\left(u=u^{*}\right)$ $\leq \frac{1}{2}$. This leads to

$$
\frac{1}{2}\left(\left(u^{*}\right)^{2}+\left(x^{*}(1)\right)^{2}\right)+\gamma\left(u^{*}\right)=\frac{(2+\beta)-(1+\alpha)^{2}}{2(2+\beta)} \leq \frac{1}{2},
$$

which is always fulfilled for $\beta>-1$. Consider

$$
\max _{\alpha, \beta} \gamma\left(u^{*}\right)=\max _{\alpha, \beta} \frac{1}{2}\left(\beta\left(\frac{1+\alpha}{2+\beta}\right)^{2}-2 \alpha \frac{1+\alpha}{2+\beta}\right)=\max _{\alpha, \beta} \frac{\beta-4 \alpha-(4+\beta) \alpha^{2}}{2(2+\beta)^{2}} .
$$

The maximization with respect to $\alpha$ leads to $\alpha=\frac{-2}{4+\beta}$, which in turn leads to

$$
\max _{\alpha, \beta} \gamma\left(u^{*}\right)=\max _{\beta} \frac{1}{2(4+\beta)} \text {. }
$$

Based upon this, the best value for $\beta$ is $\beta=-1+\epsilon$, where $\epsilon$ is an arbitrarily small positive number. Subsequently, $\alpha=-\frac{2}{3}+\frac{2}{9} \epsilon$ up to first order in $\epsilon$, and with the same accuracy, $u^{*}=-\frac{1}{3}+\frac{1}{9} \epsilon$. This leads to

$$
J_{\mathrm{F}}^{\mathrm{c}}=\frac{4}{9}-\frac{1}{27} \epsilon, \quad J_{\mathrm{L}}=\frac{1}{6}-\frac{1}{18} \epsilon,
$$

which is a best result for the bank within the class of quadratic $\gamma$ functions. Without the transaction costs for the investor, its costs would be

$$
J_{\mathrm{F}}=J_{\mathrm{F}}^{\mathrm{c}}-J_{\mathrm{L}}=\frac{5}{18}+\frac{1}{54} \epsilon,
$$

which is less than what he would have obtained by playing $u=0$. Now that $\alpha$ and $\beta$ have given values, it must be checked whether $\gamma\left(u^{*}\right)>0$ in a neighborhood of $u^{*}$. This is fortunately true. Further away from $u^{*}$, the function $\gamma$ can be adjusted such that $\gamma(\cdot) \geq 0$ everywhere.

One might be tempted to think ${ }^{1}$ that the bank can get its transaction costs arbitrarily close to $\frac{1}{4}$ by means of the following nonquadratic choice:

$$
\gamma(u)= \begin{cases}0, & \text { if } u=0 \\ \frac{1}{4}-\epsilon, & \text { if } u \neq 0\end{cases}
$$

\footnotetext{
${ }^{1}$ As was the case in [5].
} 
Here $\epsilon$ is an arbitrarily small positive number. We will consider directly the more general case

$$
\gamma(u)= \begin{cases}0, & \text { if } u=0 \\ \delta-\epsilon, & \text { if } u \neq 0\end{cases}
$$

where $\delta$ is a parameter to be chosen by the leader. Without loss of generality, we may assume that the follower's answer is of the form

$$
u= \begin{cases}0, & \text { if } 0 \leq t \leq 1-\sigma \\ v, & \text { if } 1-\sigma<t \leq 1\end{cases}
$$

for certain parameters $0 \leq \sigma \leq 1$ and $v$. With these parameters, one can easily derive that, apart from $\epsilon$-terms,

$$
J_{\mathrm{F}}^{\mathrm{c}}=\frac{1}{2} \sigma v^{2}+\frac{1}{2}(1+\sigma v)^{2}+\sigma \delta .
$$

Minimization with respect to both $v$ and $\sigma$ leads to

$$
\sigma=-1+\frac{1}{\sqrt{2 \delta}}, \quad v=\sqrt{2 \delta}, \quad J_{\mathrm{F}}^{\mathrm{c}}=-\delta+\sqrt{2 \delta}
$$

Let us now consider the leader's role. He wants to maximize $J_{\mathrm{L}}=\sigma v=\delta(-1+$ $\left.\frac{1}{\sqrt{2 \delta}}\right)$, subject to the condition $J_{\mathrm{F}}^{\mathrm{c}} \leq J_{\mathrm{F}}(u=0)=\frac{1}{2}$. If we continue to disregard the $\epsilon$-terms, this constraint is always satisfied and maximization of $J_{\mathrm{L}}$ leads to $\delta=\frac{1}{8}$, $J_{\mathrm{L}}^{*}=\frac{1}{8}, J_{\mathrm{F}}^{\mathrm{c} *}=\frac{3}{8}$. Thus, the profit of the bank turns out to be $\frac{1}{8}$, apart from an $\epsilon$-term, which is clearly less than what could be obtained with the best quadratic $\gamma$.

\subsection{Two Time Steps}

Here, we consider a discretized version of the continuous time problem. The model is

$$
x_{1}=x_{0}+\frac{1}{2} u_{1}=1+\frac{1}{2} u_{1}, \quad x_{2}=x_{1}+\frac{1}{2} u_{2}=1+\frac{1}{2}\left(u_{1}+u_{2}\right),
$$

and the criteria are

$$
\begin{aligned}
& J_{\mathrm{F}}^{\mathrm{c}}=\frac{1}{4}\left(u_{1}^{2}+u_{2}^{2}\right)+\frac{1}{2}\left(1+\frac{1}{2}\left(u_{1}+u_{2}\right)\right)^{2}+\frac{1}{2}\left(\gamma\left(u_{1}\right)+\gamma\left(u_{2}\right)\right), \\
& J_{\mathrm{L}}=\frac{1}{2}\left(\gamma\left(u_{1}\right)+\gamma\left(u_{2}\right)\right) .
\end{aligned}
$$

In Sect. 3.3, we will consider the same problem again, but then with more than 2 time steps. 


\subsubsection{First Attempt}

With the text immediately above Conjecture 2.1 in mind, a reasonable assumption seems to be that $J_{\mathrm{F}}^{\mathrm{c}}$ is minimized for the $u_{i}$-values which minimize

$$
J_{\mathrm{F}} \stackrel{\text { def }}{=} \frac{1}{4}\left(u_{1}^{2}+u_{2}^{2}\right)+\frac{1}{2}\left(1+\frac{1}{2}\left(u_{1}+u_{2}\right)\right)^{2} .
$$

These values are $u_{1}=u_{2}=-\frac{1}{2}$. Since

$$
J_{\mathrm{F}}\left(u_{1}=-\frac{1}{2}, u\right)=J_{\mathrm{F}}\left(u, u_{2}=-\frac{1}{2}\right)=\frac{3}{8} u^{2}+\frac{3}{8} u+\frac{11}{32},
$$

we try the $\gamma$-function

$$
\frac{1}{2} \gamma(u)=-\left(\frac{3}{8} u^{2}+\frac{3}{8} u\right)(1-\epsilon)
$$

with a small positive $\epsilon$. With this $\gamma$-function and with $u_{1}=u_{2}=-\frac{1}{2}$, it is easily shown that

$$
J_{\mathrm{F}}^{\mathrm{c}}=\frac{1}{4}+2(1-\epsilon) \frac{3}{32}<J_{\mathrm{F}}\left(u_{1}=0, u_{2}=0\right)=\frac{1}{2}, \quad J_{\mathrm{L}}=2(1-\epsilon) \frac{3}{32} .
$$

Please note that $\frac{1}{2}\left(J_{\mathrm{F}}\left(u_{1}=0, u_{2}=-\frac{1}{2}\right)-J_{\mathrm{F}}\left(u_{1}=-\frac{1}{2}, u_{2}=-\frac{1}{2}\right)\right)$ exactly equals $\frac{3}{32}$ which is the same fraction as which appeared in the previous formula. This is no coincidence; if the follower only played optimally during the second step, he faces a half times the total transaction costs as expressed by $J_{\mathrm{L}}$ in (6).

As it stands, with the $\gamma$ chosen, the Hessian of $J_{\mathrm{F}}$ with respect to $u_{1}$ and $u_{2}$ is not positive definite at the point $u_{i}=-\frac{1}{2}, i=1,2$, and therefore the follower can do better than choosing $u_{i}=-\frac{1}{2}, i=1,2$. To avoid this deviating behavior on part of the follower, the leader will adjust the $\gamma$-function in such a way that $u_{1}=u_{2}=-\frac{1}{2}$ is best for the follower as follows. On the interval $-\frac{1}{2} \leq u \leq 0, \gamma$ remains as given by (5). For $u<-\frac{1}{2}$, we choose a decreasing function of $u$ (i.e. increasing with $|u|$ ), which is continuous at $u=-\frac{1}{2}$, e.g.

$$
\gamma(u)=-u-\frac{1}{2}+(1-\epsilon) \frac{3}{32} .
$$

If we require the function $\gamma$ to be even, then it is defined for $u>0$ also. With this choice of $\gamma$, the best the follower can do is to choose $u_{i}=-\frac{1}{2}, i=1,2$.

Remark 3.1 A different, discontinuous and nonmonotonous choice for the leader is

$$
\gamma(u)= \begin{cases}0, & u=0 \\ \frac{1}{4}-\epsilon, & |u|=\frac{1}{2} \\ \text { large, } & \text { elsewhere }\end{cases}
$$


The reader should contemplate why this choice is not the best one. (Answer: this choice of $\gamma$ leads to $u_{1}=0, u_{2}=-\frac{1}{2}$, or the other way around, and $J_{\mathrm{F}}=\frac{15}{32}-\epsilon$ and $J_{\mathrm{L}}=\frac{1}{8}-\frac{1}{2} \epsilon$.)

\subsubsection{Second Attempt}

This is a brute force attempt and starts from the basics of what the leader might be able to achieve. Inspired by the note in the previous subsection, we (actually the leader) now try to find $\alpha$ and $\beta$ values, not to be confused with the same symbols in Sect. 3.1, that maximize

$$
J_{\mathrm{F}}(0, \beta)-J_{\mathrm{F}}(\alpha, \beta) .
$$

Because of the symmetry with respect to $u_{1}$ and $u_{2}$, an equivalent problem is to maximize

$$
J_{\mathrm{F}}(\alpha, 0)-J_{\mathrm{F}}(\alpha, \beta) .
$$

Again using the symmetry (since we can assume $u_{1}^{*}=u_{2}^{*}$ ), another equivalent problem is to maximize the sum of these two expressions,

$$
J_{\mathrm{F}}(0, \beta)-J_{\mathrm{F}}(\alpha, \beta)+J_{\mathrm{F}}(\alpha, 0)-J_{\mathrm{F}}(\alpha, \beta) .
$$

This leads to $\alpha=\beta=-\frac{2}{5}$. Subsequently, the leader chooses $\gamma(u)$ in such a way that the follower will indeed choose $\alpha=\beta=-\frac{2}{5}$. A slightly different reasoning leading to the same result is that the leader wants to maximize $\delta=\frac{1}{2}(\gamma(\alpha)+\gamma(\beta))$, with $\alpha=\beta$, subject to

$$
\begin{aligned}
& J_{\mathrm{F}}^{\mathrm{c}}(\alpha, \beta) \leq J_{\mathrm{F}}^{\mathrm{c}}(0, \beta) \rightarrow J_{\mathrm{F}}(\alpha, \beta)+\frac{1}{2} \delta \leq J_{\mathrm{F}}(0, \beta) \rightarrow \frac{1}{2} \delta \leq J_{\mathrm{F}}(0, \beta)-J_{\mathrm{F}}(\alpha, \beta), \\
& J_{\mathrm{F}}^{\mathrm{c}}(\alpha, \beta) \leq J_{\mathrm{F}}^{\mathrm{c}}(\alpha, 0) \rightarrow J_{\mathrm{F}}(\alpha, \beta)+\frac{1}{2} \delta \leq J_{\mathrm{F}}(\alpha, 0) \rightarrow \frac{1}{2} \delta \leq J_{\mathrm{F}}(\alpha, 0)-J_{\mathrm{F}}(\alpha, \beta), \\
& J_{\mathrm{F}}^{\mathrm{c}}(\alpha, \beta) \leq J_{\mathrm{F}}^{\mathrm{c}}(0,0) \rightarrow J_{\mathrm{F}}(\alpha, \beta)+\delta \leq J_{\mathrm{F}}(0,0) \rightarrow \delta \leq J_{\mathrm{F}}(0,0)-J_{\mathrm{F}}(\alpha, \beta),
\end{aligned}
$$

for suitably chosen $\alpha=\beta \neq 0$. The maximal $\delta$ is obtained for $\alpha=\beta=-\frac{2}{5}$ and thus $\delta=\frac{1}{5}$. Please note that this is a better result for the leader than the one obtained with the first attempt where $\delta=\frac{3}{16}$. Conjecture 2.1 does not hold though.

One might be tempted to think that

$$
\gamma^{*}(u)= \begin{cases}0, & \text { if } u=0 \\ \delta-\epsilon, & \text { if } u \neq 0\end{cases}
$$

with $\delta=\frac{1}{5}$ is the optimal function for the leader. This is not true. The follower, minimizing

$$
J_{\mathrm{F}}^{\mathrm{c}}=\frac{1}{4}\left(u_{1}^{2}+u_{2}^{2}\right)+\frac{1}{2}\left(1+\frac{1}{2}\left(u_{1}+u_{2}\right)\right)^{2}+\frac{1}{2}\left(\gamma^{*}\left(u_{1}+\gamma^{*}\left(u_{2}\right)\right),\right.
$$

faces three possibilities: 
1. Suppose that $u_{1}^{*} \neq 0$ and $u_{2}^{*} \neq 0$. Then, the best solution is $u_{1}^{*}=u_{2}^{*}=-\frac{1}{2}$, resulting in $J_{\mathrm{F}}^{\mathrm{c} *}=\frac{9}{20}-\epsilon$.

2. Suppose that $u_{1}^{*}=0$ and $u_{2}^{*} \neq 0$ (or the other way around). This leads to $u_{2}^{*}=-\frac{2}{3}$, resulting in $J_{\mathrm{F}}^{\mathrm{c} *}=\frac{13}{30}-\frac{1}{2} \epsilon$.

3. Suppose that $u_{1}^{*} \neq 0$ and $u_{2}^{*} \neq 0$. This results in $J_{\mathrm{F}}^{\mathrm{c} *}=\frac{1}{2}$.

The second possibility is the one to be chosen by the follower and this choice is different from $u_{1}=u_{2}=-\frac{2}{5}$ which should have been the choice of the follower, at least in the eyes of the leader. Therefore, $\gamma(u)$ should not be chosen as in (9), but rather as

$$
\begin{aligned}
\frac{1}{2} \gamma(u) & =J_{\mathrm{F}}\left(u_{1}=0, u_{2}=-\frac{2}{5}\right)-J_{\mathrm{F}}\left(u,-\frac{2}{5}\right)+\frac{1}{2} \epsilon\left[\left(u+\frac{2}{5}\right)^{2}-\frac{4}{25}\right] \\
& =-\frac{3}{8} u^{2}-\frac{2}{5} u+\frac{1}{2} \epsilon\left(u^{2}+\frac{4}{5} u\right) .
\end{aligned}
$$

The first part at this right-hand side makes $J_{\mathrm{F}}\left(u,-\frac{2}{5}\right)+\frac{1}{2} \gamma(u)$ a constant for varying $u$; the second part, the term with $\epsilon$ yields a unique minimum for $u=-\frac{2}{5}$ and hence this $u$ will be the follower's choice.

Unfortunately, this is not the whole story. The Hessian of

$$
\begin{aligned}
J_{\mathrm{F}}^{\mathrm{c}}\left(u_{1}, u_{2}\right)= & \frac{1}{4}\left(u_{1}^{2}+u_{2}^{2}\right)+\frac{1}{2}\left(1+\frac{1}{2}\left(u_{1}+u_{2}\right)\right)^{2} \\
& +\sum_{i=1}^{2}\left(-\frac{3}{8} u_{i}^{2}-\frac{2}{5} u_{i}+\frac{1}{2} \epsilon\left(u_{i}^{2}+\frac{4}{5} u_{i}\right)\right)
\end{aligned}
$$

equals

$$
\left(\begin{array}{ll}
\epsilon & \frac{1}{4} \\
\frac{1}{4} & \epsilon
\end{array}\right)
$$

and is not positive definite for $\epsilon<\frac{1}{4}$. For such $\epsilon$, the situation can be remedied by choosing a different $\gamma(u)$ on the interval $u<-\frac{2}{5}$, in the same way as this was done in Sect. 3.2.1.

If monotonicity of $\gamma$ with respect to $|u|$, tacitly assumed above, is not required, then another candidate strategy for the leader is

$$
\gamma(u)= \begin{cases}0, & u=0, \\ \frac{1}{5}-\epsilon, & |u|=\frac{2}{5}, \\ \text { large, } & \text { elsewhere, }\end{cases}
$$

which turns out to be optimal as well. 


\subsection{Many Time Steps and Limit to Infinity}

In this subsection, we investigate the solution if more than two time steps are used to approximate the original continuous time problem and what happens if the number of time steps approaches infinity. Will in the latter case the solution become identical to the one of Sect. 3.1? We consider the model

$$
x_{i}=x_{i-1}+\frac{1}{N} u_{i}, \quad i=1,2, \ldots, N, \quad x_{0}=1,
$$

and the criteria

$$
J_{\mathrm{F}}=\frac{1}{2 N} \sum_{i=1}^{N} u_{i}^{2}+\frac{1}{2}\left(1+\frac{1}{N} \sum_{i=1}^{N} u_{i}\right)^{2}, \quad J_{\mathrm{L}}=\frac{1}{N} \sum_{i=1}^{N} \gamma\left(u_{i}\right) .
$$

The equivalent expression for (8) becomes

$$
\begin{aligned}
\frac{1}{2} & {\left[\frac{1}{N} \sum_{k=1}^{N}\left(\sum_{i=1, i \neq k}^{N} u_{i}^{2}\right)+\sum_{k=1}^{N}\left(1+\frac{1}{N} \sum_{i=1, i \neq k}^{N} u_{i}\right)^{2}\right] } \\
& -\frac{1}{2} \sum_{i=1}^{N} u_{i}^{2}-\frac{1}{2} N\left(1+\frac{1}{N} \sum_{i=1}^{N} u_{i}\right)^{2} .
\end{aligned}
$$

The derivative of this expression with respect to $u_{1}$ equals zero. Substitution of $u_{2}=$ $\cdots=u_{N}=u_{1}$ subsequently leads to

$$
u_{i}^{*}=-\frac{N}{3 N-1}, \quad i=1,2, \ldots, N .
$$

For $N=2$, this coincides with the results of the previous subsection. For $N \rightarrow \infty$, we get $u_{i}^{*}=-\frac{1}{3}, i=1,2, \ldots, N$. It is easily shown that the profit for the bank is $\frac{N}{2(3 N-1)}$. Note that, for $N \rightarrow \infty$, this profit equals $\frac{1}{6}$, which equals the result obtained with the best quadratic $\gamma$-function.

Let us consider $J_{\mathrm{F}}$ as a function of $u_{1}$ only and with $u_{2}=\cdots=u_{N}=-\frac{N}{3 N-1}$,

$$
J_{\mathrm{F}}\left(u_{1}, *\right)=\frac{1}{2}\left[\frac{1}{N} u_{1}^{2}+\frac{N-1}{N}\left(\frac{N}{3 N-1}\right)^{2}+\left(1+\frac{1}{N}\left(u_{1}-\frac{N(N-1)}{3 N-1}\right)\right)^{2}\right],
$$

where $*$ is a short-hand notation for $u_{2 \leq i \leq N}=-\frac{N}{3 N-1}$. For this function,

$$
\begin{aligned}
& J_{\mathrm{F}}\left(u_{1}=0, *\right)-J_{\mathrm{F}}\left(u_{1}=-\frac{N}{3 N-1}, *\right) \\
& =\frac{1}{2}\left[\frac{N(N-1)+(2 N)^{2}}{(3 N-1)^{2}}-\frac{N^{2}+(2 N-1)^{2}}{(3 N-1)^{2}}\right],
\end{aligned}
$$

which is the $N$-equivalent of (7). If we calculate $N\left(J_{\mathrm{F}}\left(u_{1}=0, *\right)-J_{\mathrm{F}}\left(u_{1}=\right.\right.$ $\left.\left.-\frac{N}{3 N-1}, *\right)\right)$, the result is $\frac{N}{2(3 N-1)}$, which equals the profit of the bank (as already 
obtained earlier). Apart from an $\epsilon$ term, it is necessary from the leader's point of view that

$$
J_{\mathrm{F}}\left(u_{1}=-\frac{N}{3 N-1}, *\right)+\frac{1}{N} \gamma \leq J_{\mathrm{F}}\left(u_{1}=0, *\right),
$$

or with a quadratic $\epsilon$ term,

$$
J_{\mathrm{F}}+\frac{1}{N} \gamma=J_{\mathrm{F}}\left(u_{1}=0, *\right)+\frac{1}{N} \epsilon\left[\left(u_{1}+\frac{N}{3 N-1}\right)^{2}-\left(\frac{N}{3 N-1}\right)^{2}\right] .
$$

Hence,

$$
\begin{aligned}
\frac{1}{N} \gamma\left(u_{1}\right)= & \frac{1}{2}\left[\frac{N(N-1)+(2 N)^{2}}{(3 N-1)^{2}}-\frac{1}{N} u_{1}^{2}-\frac{N-1}{N}\left(\frac{N}{3 N-1}\right)^{2}\right. \\
& \left.-\left(1+\frac{1}{N}\left(u_{1}-\frac{N(N-1)}{3 N-1}\right)\right)^{2}\right]+\frac{\epsilon}{N}\left(u_{1}^{2}+\frac{2 N}{3 N-1} u_{1}\right) \\
= & \frac{1}{2}\left[-\frac{1}{N} u_{1}^{2}-\frac{1}{N^{2}} u_{1}^{2}-\frac{4}{3 N-1} u_{1}\right]+\frac{\epsilon}{N}\left(u_{1}^{2}+\frac{2 N}{3 N-1} u_{1}\right) .
\end{aligned}
$$

For $N \rightarrow \infty$, this leads to exactly the optimal quadratic function obtained before. This is at least true for $\epsilon=0$. The terms linear in $\epsilon$ differ, however. We now write

$$
\begin{aligned}
J_{\mathrm{F}}^{\mathrm{c}}\left(u_{1}, \ldots, u_{N}\right)= & J_{\mathrm{F}}\left(u_{1}, \ldots, u_{N}\right)+\frac{1}{2}\left[\sum_{i=1}^{N}\left(-\frac{1}{N} u_{i}^{2}-\frac{1}{N^{2}} u_{i}^{2}-\frac{2}{3 N-1} u_{i}\right)\right. \\
& \left.+\frac{2 \epsilon}{N}\left(u_{i}^{2}+\frac{4 N}{3 N-1} u_{i}\right)\right] .
\end{aligned}
$$

The Hessian equals

$$
\left(\begin{array}{cccc}
\frac{2 \epsilon}{N} & \frac{1}{N^{2}} & \cdots & \frac{1}{N^{2}} \\
\frac{1}{N^{2}} & \ddots & \ddots & \vdots \\
\vdots & \ddots & \ddots & \frac{1}{N^{2}} \\
\frac{1}{N^{2}} & \cdots & \frac{1}{N^{2}} & \frac{2 \epsilon}{N}
\end{array}\right)
$$

For $N>\frac{1}{2 \epsilon}$, all eigenvalues lie in the right half plane. For $N \leq \frac{1}{2 \epsilon}$, however, the Hessian is not positive definite. In the latter case, one uses the trick of Sect. 3.2.1, i.e. for $-\frac{N}{3 N-1} \leq u \leq 0, \gamma(u)$ is as above, and for $u<-\frac{N}{3 N-1}$ we choose it as a decreasing function.

\section{Second Dynamic Example}

The example treated in Sect. 3 is relatively simple in the sense that the optimal $u$ is a constant, both for the continuous time and the discrete time problems. The example 
of this section is more complicated in the sense that the optimal $u$ varies with time. The starting point is the dynamic model

$$
\dot{x}=u, \quad x(0)=1,
$$

with criterion

$$
\min _{u} \frac{1}{2} \int_{0}^{1}\left(x^{2}+u^{2}\right) \mathrm{d} t+\frac{1}{2} x^{2}(1) .
$$

An essential difference with the problem of the previous section is that the optimal control is not constant anymore: $u^{*}(t)=-e^{-t}$ which leads to the minimal value $J_{\mathrm{F}}^{\mathrm{c} *}=\frac{1}{2}$. In the discretized problems (see the coming subsections) we cannot expect all $u_{i}^{*}$ to be equal anymore. Consequently $\gamma(u)$ will have to be specified in the neighbourhood of these different $u_{i}^{*}$-values.

The best quadratic $\gamma$ can be calculated as before for the other example. As in Sect. 3 we try $\gamma(u)=\frac{1}{2} \beta u^{2}+\alpha u$. The value function, to be minimized with respect to $\alpha$ and $\beta$, is (assuming that $x(0)=1$ )

$$
\frac{1}{2} S(0)+k(0)+m(0)
$$

where $S(t), k(t)$ and $m(t)$ satisfy, see [2],

$$
\begin{aligned}
& \dot{S}=\frac{S^{2}}{1+\beta}-1, \quad S(1)=1, \\
& \dot{k}=\frac{S}{1+\beta}(k+\alpha), \quad k(1)=0, \\
& \dot{m}=\frac{1}{1+\beta}\left(k \alpha+\frac{1}{2} k^{2}\right), \quad m(1)=0 .
\end{aligned}
$$

It does not seem a very appetizing exercise to work this out analytically.

\subsection{Two Time Steps}

The model is as before, (4). The criterion for the leader also remains the same, but the criterion for the follower is now defined as

$$
\begin{aligned}
J_{\mathrm{F}}^{\mathrm{c}}= & \frac{1}{4}\left(u_{1}^{2}+u_{2}^{2}+x_{0}^{2}+x_{1}^{2}\right)+\frac{1}{2} x_{2}^{2}+\frac{1}{2}\left(\gamma\left(u_{1}\right)+\gamma\left(u_{2}\right)\right) \\
= & \frac{1}{4}\left(u_{1}^{2}+u_{2}^{2}+1+\left(1+\frac{1}{2} u_{1}\right)^{2}\right)+\frac{1}{2}\left(1+\frac{1}{2}\left(u_{1}+u_{2}\right)\right)^{2} \\
& +\frac{1}{2}\left(\gamma\left(u_{1}\right)+\gamma\left(u_{2}\right)\right) \\
= & \frac{7}{16} u_{1}^{2}+\frac{3}{8} u_{2}^{2}+1+\frac{3}{4} u_{1}+\frac{1}{2} u_{2}+\frac{1}{4} u_{1} u_{2}+\frac{1}{2}\left(\gamma\left(u_{1}\right)+\gamma\left(u_{2}\right)\right) .
\end{aligned}
$$


The best the leader can hope for is the maximum value of $\frac{1}{2}\left(\delta_{1}+\delta_{2}\right)$, where $\delta_{1}=$ $\gamma\left(u_{1}\right)$ and $\delta_{2}=\gamma\left(u_{2}\right)$, for which not only the following inequalities must hold for a suitable choice of $\alpha$ and $\beta$ :

$$
\begin{aligned}
& J_{\mathrm{F}}(\alpha, \beta)+\frac{1}{2}\left(\delta_{1}+\delta_{2}\right) \leq J_{\mathrm{F}}(0, \beta)+\frac{1}{2} \delta_{2}, \\
& J_{\mathrm{F}}(\alpha, \beta)+\frac{1}{2}\left(\delta_{1}+\delta_{2}\right) \leq J_{\mathrm{F}}(\alpha, 0)+\frac{1}{2} \delta_{1}, \\
& J_{\mathrm{F}}(\alpha, \beta)+\frac{1}{2}\left(\delta_{1}+\delta_{2}\right) \leq J_{\mathrm{F}}(0,0),
\end{aligned}
$$

but also

$$
\begin{aligned}
& J_{\mathrm{F}}(\alpha, \beta)+\frac{1}{2}\left(\delta_{1}+\delta_{2}\right) \leq J_{\mathrm{F}}(\beta, \beta)+\delta_{2}, \\
& J_{\mathrm{F}}(\alpha, \beta)+\frac{1}{2}\left(\delta_{1}+\delta_{2}\right) \leq J_{\mathrm{F}}(\alpha, \alpha)+\delta_{1}, \\
& J_{\mathrm{F}}(\alpha, \beta)+\frac{1}{2}\left(\delta_{1}+\delta_{2}\right) \leq J_{\mathrm{F}}(0, \alpha)+\frac{1}{2} \delta_{1}, \\
& J_{\mathrm{F}}(\alpha, \beta)+\frac{1}{2}\left(\delta_{1}+\delta_{2}\right) \leq J_{\mathrm{F}}(\beta, 0)+\frac{1}{2} \delta_{2}, \\
& J_{\mathrm{F}}(\alpha, \beta)+\frac{1}{2}\left(\delta_{1}+\delta_{2}\right) \leq J_{\mathrm{F}}(\beta, \alpha)+\frac{1}{2}\left(\delta_{1}+\delta_{2}\right) .
\end{aligned}
$$

Together these inequalities, which are only necessary conditions, lead to

$$
\begin{aligned}
\delta_{1} & \leq-\frac{7}{8} \alpha^{2}-\frac{3}{2} \alpha-\frac{1}{2} \alpha \beta, \\
\delta_{2} & \leq-\frac{3}{4} \beta^{2}-\beta-\frac{1}{2} \alpha \beta, \\
\delta_{1}+\delta_{2} & \leq-\frac{7}{8} \alpha^{2}-\frac{3}{2} \alpha-\frac{3}{4} \beta^{2}-\beta-\frac{1}{2} \alpha \beta, \\
\delta_{1}-\delta_{2} & \leq-\frac{7}{8} \alpha^{2}+\frac{11}{8} \beta^{2}-\frac{3}{2} \alpha+\frac{3}{2} \beta-\frac{1}{2} \alpha \beta, \\
\delta_{2}-\delta_{1} & \leq+\frac{5}{4} \alpha^{2}-\frac{3}{4} \beta^{2}+\alpha-\beta-\frac{1}{2} \alpha \beta, \\
\delta_{2} & \leq-\frac{1}{8} \alpha^{2}-\frac{3}{4} \beta^{2}-\frac{1}{2} \alpha-\beta-\frac{1}{2} \alpha \beta, \\
\delta_{1} & \leq-\frac{7}{8} \alpha^{2}+\frac{1}{8} \beta^{2}-\frac{3}{2} \alpha-\frac{5}{2} \beta-\frac{1}{2} \alpha \beta, \\
0 & \leq-\frac{1}{16} \alpha^{2}+\frac{1}{16} \beta^{2}-\frac{1}{4} \alpha+\frac{1}{4} \beta .
\end{aligned}
$$

Guided by Sect. 3, one might assume that the first two inequalities determine the maximizing $\delta_{i}$. A simple calculation shows that this is not true unfortunately. $\mathrm{Nu}-$ 
merically it was found that the solution, satisfying all inequalities, is $\delta_{1}=\frac{9}{14}, \delta_{2}=0$, $\alpha=-\frac{6}{7}$ and $\beta=0$. Generally we should have $\beta \neq 0$, but $\delta_{2}=0$ makes $\beta=0$ a valid answer.

\subsection{Three Time Steps}

The model is

$$
\begin{aligned}
& x_{1}=1+\frac{1}{3} u_{1}, \\
& x_{2}=1+\frac{1}{3}\left(u_{1}+u_{2}\right), \\
& x_{3}=1+\frac{1}{3}\left(u_{1}+u_{2}+u_{3}\right),
\end{aligned}
$$

and the criterion is

$$
\begin{aligned}
J_{\mathrm{F}}^{\mathrm{c}}= & J_{\mathrm{F}}\left(u_{1}, u_{2}, u_{3}\right)+\frac{1}{3} \sum_{i=1}^{3} \delta_{i}=\frac{1}{2} \sum_{i=1}^{3} \frac{1}{3}\left(x_{i-1}^{2}+u_{i}^{2}\right)+\frac{1}{2} x_{3}^{2}+\frac{1}{3} \sum_{i=1}^{3} \delta_{i} \\
= & \frac{1}{3}\left(\frac{7}{9} u_{1}^{2}+\frac{13}{18} u_{2}^{2}+\frac{2}{3} u_{3}^{2}+\frac{5}{3} u_{1}+\frac{4}{3} u_{2}+u_{3}+\frac{4}{9} u_{1} u_{2}+\frac{1}{3} u_{1} u_{3}+\frac{1}{3} u_{2} u_{3}+3\right. \\
& \left.+\sum_{i=1}^{3} \delta_{i}\right),
\end{aligned}
$$

where $x_{0}=1$ and $\delta_{i}=\gamma\left(u_{i}\right)$. The leader now wants to maximize $\frac{1}{3} \sum_{i=1}^{3} \delta_{i}$ subject to

$$
J_{\mathrm{F}}(\alpha, \beta, \gamma)+\frac{1}{3} \sum_{i=1}^{3} \delta_{i} \leq J_{\mathrm{F}}(\arg 1, \arg 2, \arg 3)+\frac{1}{3}\left(\text { combination of some } \delta^{\prime} \mathrm{s}\right),
$$

where $\arg i, i=1,2,3$, can be any of the values $0, \alpha, \beta$, or $\gamma$. For an argument $\alpha, \delta_{1}$ is added to "the combination of some" $\delta$ 's; similarly for an argument $\beta, \delta_{2}$ is added, and for an argument $\gamma, \delta_{3}$ is added. For the argument 0 , nothing is added to the righthand side. Altogether we get 63 inequalities (actually 64, but one inequality is the identity). An optimum (possibly a local one) found numerically is $\delta_{1}=0.893, \delta_{2}=$ $\delta_{3}=0, \alpha=-1.071, \beta=\gamma=0 .^{2}$ In fractions, this is probably $\delta_{1}=\frac{25}{28}, \alpha=-\frac{15}{14}$.

\subsection{Many Time Steps and Limit to Infinity}

The model is

$$
x_{i}=x_{i-1}+\frac{1}{N} u_{i}, \quad i=1,2, \ldots, N, x_{0}=1
$$

\footnotetext{
${ }^{2}$ Etienne de Klerk (Delft) is acknowledged for these numerical calculations and those of the previous subsection.
} 
and the criteria are

$$
\begin{aligned}
J_{\mathrm{F}} & =\frac{1}{2 N} \sum_{i=1}^{N}\left(u_{i}^{2}+x_{i-1}^{2}\right)+\frac{1}{2} x_{N}^{2} \\
& =\frac{1}{2 N} \sum_{i=1}^{N}\left(u_{i}^{2}+\left(1+\frac{1}{N} \sum_{k=1}^{i-1} u_{k}\right)^{2}\right)+\frac{1}{2}\left(1+\frac{1}{N} \sum_{i=1}^{N} u_{i}\right)^{2}, \\
J_{\mathrm{L}} & =\frac{1}{N} \sum_{i=1}^{N} \gamma\left(u_{i}\right) .
\end{aligned}
$$

First, we want to solve $\min _{u} J_{\mathrm{F}}$ subject to the model equations. This leads to a linear equation in $u$,

$$
\left(\begin{array}{ccccc}
d+\zeta_{1} & \zeta_{2} & \zeta_{3} & \ldots & \zeta_{N} \\
\zeta_{2} & d+\zeta_{2} & \zeta_{3} & & \vdots \\
\zeta_{3} & \zeta_{3} & d+\zeta_{3} & & \vdots \\
\vdots & & & \ddots & \zeta_{N} \\
\zeta_{N} & \ldots & \ldots & \zeta_{N} & d+\zeta_{N}
\end{array}\right)\left(\begin{array}{l}
u_{1} \\
u_{2} \\
u_{3} \\
\vdots \\
u_{N}
\end{array}\right)=-N\left(\begin{array}{l}
\zeta_{1} \\
\zeta_{2} \\
\zeta_{3} \\
\vdots \\
\zeta_{N}
\end{array}\right)
$$

where

$$
d=\frac{1}{N}, \quad \zeta_{i}=\frac{1}{N^{3}}(N-i)+\frac{1}{N^{2}} .
$$

A numerical exercise indicates that the solution $u$ converges towards $-e^{-t}$, as it should, as $N \rightarrow \infty$. An upper bound for what the leader can hope for is obtained via the calculation of the maximum (with respect to $u_{i}, i=1,2, \ldots, N$ ) of

$$
\begin{aligned}
& J_{\mathrm{F}}\left(0, u_{2}, u_{3}, u_{4}, \ldots, u_{N}\right)-J_{\mathrm{F}}\left(u_{1}, u_{2}, u_{3}, \ldots, u_{N}\right) \\
& \quad+J_{\mathrm{F}}\left(u_{1}, 0, u_{3}, u_{4}, \ldots, u_{N}\right)-J_{\mathrm{F}}\left(u_{1}, u_{2}, u_{3}, \ldots, u_{N}\right) \\
& \quad+J_{\mathrm{F}}\left(u_{1}, u_{2}, 0, u_{4}, \ldots, u_{N}\right)-J_{\mathrm{F}}\left(u_{1}, u_{2}, u_{3}, \ldots, u_{N}\right) \\
& \quad \vdots \\
& \quad+J_{\mathrm{F}}\left(u_{1}, u_{2}, u_{3}, \ldots, u_{N-1}, 0\right)-J_{\mathrm{F}}\left(u_{1}, u_{2}, u_{3}, \ldots, u_{N}\right),
\end{aligned}
$$

(compare the addition of (10) and (11)) provided that all the optimizing $u_{i}$-values are different. Written out, this expression becomes

$$
\begin{gathered}
-\left(\frac{1}{2 N}+\frac{1}{2 N^{2}}\right) \sum_{l=1}^{N} u_{l}^{2}-\frac{1}{2 N} \sum_{l=1}^{N} \sum_{i=l+1}^{N}\left(\frac{1}{N^{2}} u_{l}^{2}+\frac{2}{N^{2}} u_{l} \sum_{k=1, \neq l}^{i-1} u_{k}\right) \\
-\frac{1}{N^{2}} \sum_{l=1}^{N}\left(u_{l} \sum_{i=1, \neq l}^{N} u_{i}\right)-\frac{1}{2 N} \sum_{l=1}^{N} \sum_{i=l+1}^{N} \frac{2}{N} u_{l}-\frac{1}{N} \sum_{l=1}^{N} u_{l} .
\end{gathered}
$$


Differentiation with respect to $j$ leads to

$$
\begin{aligned}
& -\left(\frac{1}{N}+\frac{1}{N^{2}}\right) u_{j}-\frac{2}{N^{2}} \sum_{l=1, l \neq j}^{N} u_{l}-\frac{1}{2 N}\left\{2 \frac{N-j}{N^{2}} u_{j}+\frac{4}{N} \sum_{l=1}^{N} c_{l, j} u_{l}\right\} \\
& -\left(\frac{1}{N}+\frac{N-j}{N^{2}}\right)=0
\end{aligned}
$$

where

$$
c_{l, j}= \begin{cases}0, & \text { if } l=j, \\ N-\max \{j, l\}, & \text { otherwise. }\end{cases}
$$

For $j=1,2, \ldots, N$, these equations can be written as

$$
\left(\begin{array}{ccccc}
d+\zeta_{1} & 2 \zeta_{2} & 2 \zeta_{3} & \ldots & 2 \zeta_{N} \\
2 \zeta_{2} & d+\zeta_{2} & 2 \zeta_{3} & & \vdots \\
2 \zeta_{3} & 2 \zeta_{3} & d+\zeta_{3} & & \vdots \\
\vdots & & & \ddots & 2 \zeta_{N} \\
2 \zeta_{N} & \ldots & \ldots & 2 \zeta_{N} & d+\zeta_{N}
\end{array}\right)\left(\begin{array}{l}
u_{1} \\
u_{2} \\
u_{3} \\
\vdots \\
u_{N}
\end{array}\right)=-N\left(\begin{array}{l}
\zeta_{1} \\
\zeta_{2} \\
\zeta_{3} \\
\vdots \\
\zeta_{N}
\end{array}\right)
$$

with $d$ and $\zeta_{i}$ defined as in (13). If this linear system of equations is symbolically written as $\left(\frac{1}{N} I+A\right) u=-N \zeta, I$ being the identity matrix, then $u=-(I-N A+$ $\left.(N A)^{2}-\cdots\right) N^{2} \zeta$. Some numerical exercises show that the solution $u$ resembles the one of (12), i.e. the values are negative, $|u(i)|$ decreases with increasing $i$. The two solutions are clearly different though.

Above, it was assumed that all $u_{i}$-values were different (which also followed from numerical evidence). In case of all $u_{i}$-values being equal, an upper bound for the best the leader can hope for is the maximum value of $\delta$ for which the following inequalities hold for a suitable choice of the $\alpha_{i}$ 's (all being equal):

$$
\begin{aligned}
& J_{\mathrm{F}}\left(\alpha_{1}, \alpha_{2}, \ldots, \alpha_{N}\right)+\frac{i}{N} \delta<J_{\mathrm{F}}\left(i \alpha^{\prime} \text { s are zero, all combinations }\right), \\
& \quad i=1,2, \ldots, N
\end{aligned}
$$

In general, the leader will have to deal with a mixture of the extreme cases (14) and (16) since he will not know ahead of time what yields the best results for him. Suppose that all optimal $u_{i}$ 's are different. Then choose $\left.\gamma_{(} u_{i}\right)=J_{\mathrm{F}}\left(u_{1}, \ldots, u_{i-1}, 0\right.$, $\left.u_{i+1}, \ldots, u_{N}\right)-J_{\mathrm{F}}\left(u_{1}, u_{2}, u_{3}, \ldots, u_{N}\right)-\epsilon$. And, for all other values of $u$, choose $\gamma$ large (except for $\gamma(0)=0$ ). In general, this function will not be monotone with respect to $|u|$ and its "usefulness" seems questionable for $N \rightarrow \infty$.

\section{Conclusions}

In [1] it was concluded that inverse Stackelberg problems are very hard to analyze and in that paper only static problems were considered. That conclusion remains valid for 
the dynamic problems of the current paper. One of the reasons to study dynamic problems in this context is that they seem to show up in reality more often.

Open Access This article is distributed under the terms of the Creative Commons Attribution Noncommercial License which permits any noncommercial use, distribution, and reproduction in any medium, provided the original author(s) and source are credited.

\section{References}

1. Olsder, G.J.: Phenomena in inverse Stackelberg games, part 1: Static problems. J. Optim. Theory Appl. (2009). doi:10.1007/s10957-009-9573-9

2. Başar, T., Olsder, G.J.: Dynamic Noncooperative Game Theory. SIAM, Philadelphia (1999)

3. Ho, Y.-C., Luh, P.B., Olsder, G.J.: A control-theoretic view on incentives. Automatica 18, 167-179 (1982)

4. Bernhard, P.: A robust control approach to option pricing including transaction costs. In: Nowak, A. (ed.) Advances in Dynamic Gases, Annals of the ISDG, vol. 7, pp. 391-416. Birkhäuser, Basel (2002)

5. Olsder, G.J.: Differential game-theoretic thoughts on option pricing and transaction costs. Int. Game Theory Rev. 2, 209-228 (2000)

6. Kuczma, M.: Functional Equations in a Single Variable. Polish Sci., Warsaw (1968)

7. Bagchi, A.: Stackelberg Differential Games in Economic Models. Lecture Notes in Control and Information Sciences, vol. 64. Springer, Berlin (1984)

8. Gradus, R.H.J.M.: The derivation of feedback Stackelberg equilibria in dynamic economic games. In: Dynamic Games in Economic Analysis. Lecture Notes in Control and Information Sciences, vol. 157, pp. 193-203. Springer, Berlin (1991)

9. He, X., Prasad, A., Sethi, S., Gutierrez, G.: A survey of Stackelberg differential game models in supply and marketing channels. J. Syst. Sci. Syst. Eng. 16(4), 385-413 (2007)

10. Bryson, A.E., Ho, Y.-C.: Applied Optimal Control. Ginn, Needham Heights (1969) 